Supporting Information

\title{
Three-Dimensional Mass Spectrometric Imaging of Biological Structures Using a Vacuum Compatible Microfluidic Device
}

\author{
Wenxiao Guo, ${ }^{\mathrm{a}, \mathrm{b}}$ Michal Kanski, ${ }^{\mathrm{c}}$ Wen Liu, ${ }^{\mathrm{a}}$ Mikołaj Gołuński, ${ }^{\mathrm{c}}$ Yadong Zhou, ${ }^{\mathrm{a}}$ Yining \\ Wang, ${ }^{\mathrm{d}}$ Cuixia Cheng, ${ }^{\mathrm{a}}$ Yingge Du, ${ }^{\mathrm{d}}$ Zbigniew Postawa, ${ }^{\mathrm{c}^{*}}$ Wei David Wei, ${ }^{\text {** }}$ and Zihua \\ $\mathrm{Zhu}^{\mathrm{a},{ }^{*}}$ \\ a Environmental Molecular Sciences Laboratory, Pacific Northwest National Laboratory, Richland, WA \\ 99354, United States \\ b Department of Chemistry and Center for Catalysis, University of Florida, Gainesville, Florida 32611, \\ United States \\ c Smoluchowski Institute of Physics, Jagiellonian University, S. Lojasiewicza 11, Kraków, Poland \\ d Physical and Computational Sciences Directorate, Pacific Northwest National Laboratory, Richland, WA \\ 99354, United States \\ *E-mail: zihua.zhu@pnnl.gov \\ *E-mail: wei@chem.ufl.edu \\ *E-mail: zbigniew.postawa@uj.edu.pl
}

\section{Table of Contents}

Calculation of the number of ice molecules detected per Ar cluster ion.........................S2

More details about the Ar cluster ion beam.................................................................S4

The reproducibility of depth profiling measurement of ice........................................S4

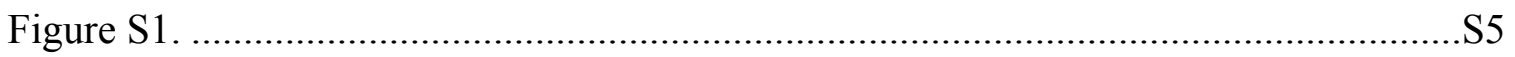

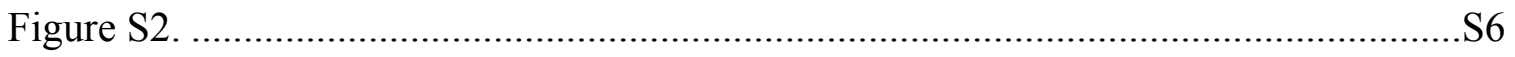

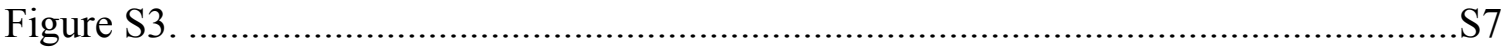




\section{Calculation of the number of ice molecules detected per Ar cluster ion}

In the sputtering process, a $20 \mathrm{keV}$ Ar cluster ion beam was used as the primary ion beam. The number of Ar cluster ions hitting the sample, $N_{\text {ion }}$, was estimated from the beam current $I$, and the sputter time $t$, as shown in equation (1):

$$
N_{i o n}=\frac{I \times t}{q}
$$

$N_{\text {ion }}:$ the number of primary ions

$q$ : the elementary charge

I: beam current

$t$ : sputter time

After a period of sputtering, the Ar cluster ion beam punched through the silicon nitride (SiN) membrane window and created a small hole that exposed the ice sample. The Ar cluster ion beam then interacted with the ice sample through the small hole, and ice molecules began to be detected. The total number of ice molecules detected, $N_{\text {ice }}$, was estimated using equation (2), where $\rho_{\text {ice}}, V_{\text {ice, }}$ and $M_{\text {ice }}$ represent the density of ice, the volume of ice sputtered off, and the molar mass of ice, respectively.

$$
N_{\text {ice }}=\frac{\rho_{\text {ice }} \times V_{\text {ice }}}{M_{\text {ice }}} \times N_{A}
$$

$N_{\text {ice }}:$ the number of ice molecules detected

$\rho_{\text {ice }}$ : the density of ice

$V_{i c e}:$ the volume of ice sputtered off

$M_{\text {ice }}:$ the molar mass of ice

$N_{A}$ : Avogadro's constant

Hence, by combining equations (1) and (2), we expressed the number of ice molecules detected per Ar cluster ion, $N$, using equation (3):

$$
N=\frac{N_{\text {ice }}}{N_{\text {ion }}}=\frac{\rho_{\text {ice }} \times V_{\text {ice }} \times N_{A}}{M_{\text {ice }} \times I \times t} \times q
$$

The primary ion beam was focused on the surface of ice sample. The scanning time was recorded in $\mathrm{s}$ and the corresponding beam current was recorded in $\mathrm{nA}$. According to equation (1), we rewrote the equation for estimating the number of primary ions $\left(N_{i o n}\right)$ reaching the sample using equation (4): 


$$
N_{\text {ion }}=\frac{10^{-9} \times I \times t}{q} \approx 0.625 \times 10^{10} \times I \times t
$$

\section{Unit of $t: \mathrm{s}$}

\section{Unit of $I: \mathrm{nA}$}

During the period of beam scanning, the ice sample was exposed and sputtered. The volume of ice molecules being sputtered off was by convention recorded in $\mu \mathrm{m}^{3}$. Thus, the equation for calculating the quantity of ice being sputtered off was rewritten as equation (5):

$$
N_{\text {ice }}=\frac{10^{-6} \times \rho_{\text {ice }} \times V_{\text {ice }}}{M_{\text {ice }}} \times N_{A} \approx 0.33 \times 10^{11} \times V_{\text {ice }}
$$

$$
\text { Unit of } V_{\text {ice }}: \mu \mathrm{m}^{3}
$$

Subsequently, the number of ice molecules detected for each individual primary Ar ion was obtained from equation (6):

$$
N=\frac{N_{\text {ice }}}{N_{\text {ion }}} \approx 5.28 \frac{V_{\text {ice }}}{\text { It }}
$$

Unit of $t: \mathrm{s}$

Unit of $I: \mathrm{nA}$

Unit of $V_{\text {ice }}: \mu \mathrm{m}^{3}$ 


\section{More details about the Ar cluster ion beam}

A $20 \mathrm{keV} \mathrm{Ar}_{1800}{ }^{+}$cluster ion beam was used as the sputtering ion beam in this work. The nominal Ar cluster size was 1800, and a wide distribution (1800 \pm 400$)$ was observed for the ion beam used in this research.

\section{The reproducibility of depth profiling measurement of ice}

The sputter rate results were reasonably reproducible when $50 \mu \mathrm{m}$ deep devices were used. We tested four $50 \mu \mathrm{m}$ devices in the first batch, and three of them produced sputter rates within the range of 1400-1600 (water molecules per $20 \mathrm{keV}$ Ar cluster ion). After a few months, we (another student) tested three devices again, and two of them yielded sputter rates within1400-1600. Therefore, the reproducibility of the $50 \mu \mathrm{m}$ device was reasonably high. 


\section{Supplementary Figures}
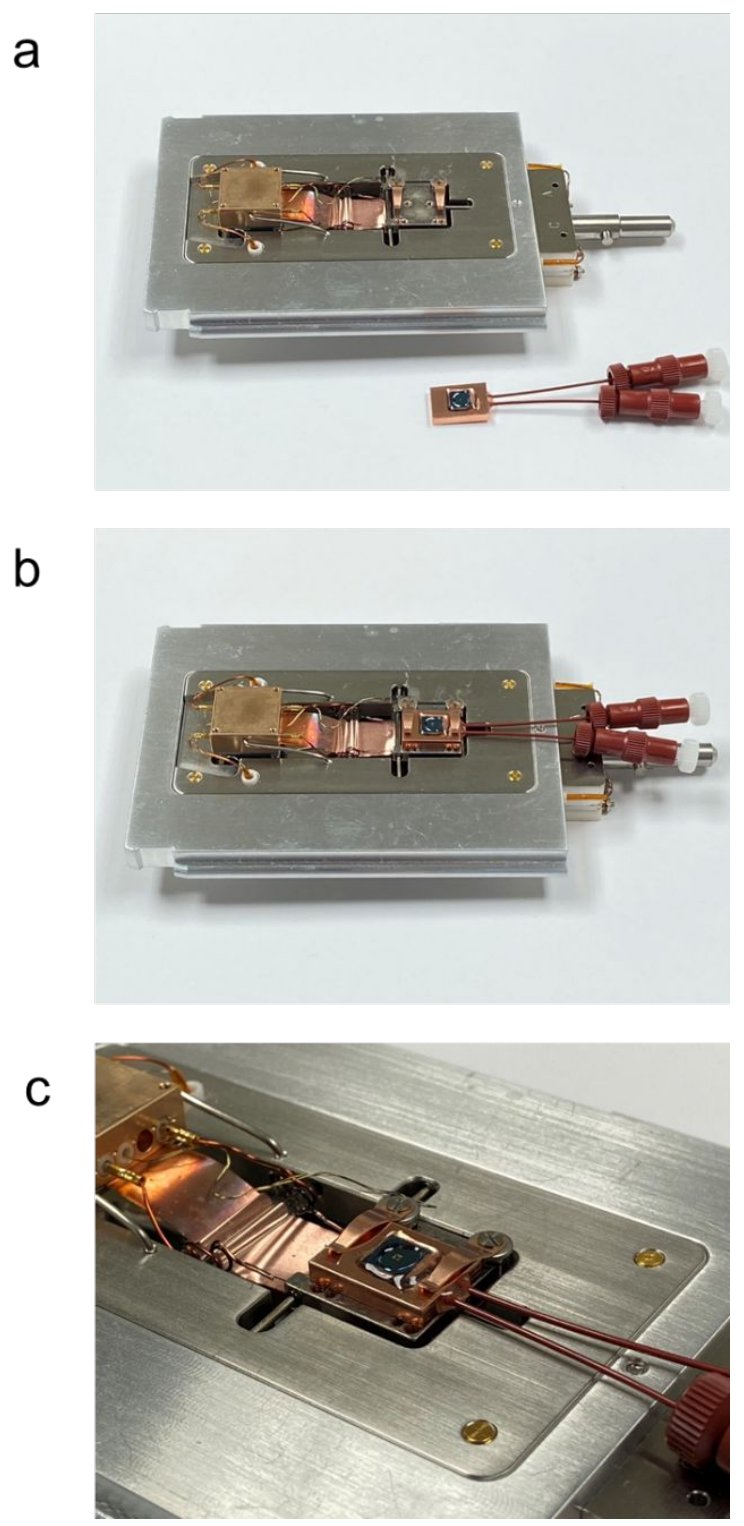

Figure S1. Images of a vacuum-compatible device for 3D molecular imaging of freezing samples using ToF-SIMS. (a) A temperature-controllable sample stage (top, directly purchased from IONTOF GmbH, the ToF-SIMS manufacturer) and a liquid cell equipped with inlet and outlet for liquids (bottom, developed in this work). (b) The assembly of the temperature-controllable stage and the liquid cell. (c) An enlarged image for the liquid cell in the assembly. 

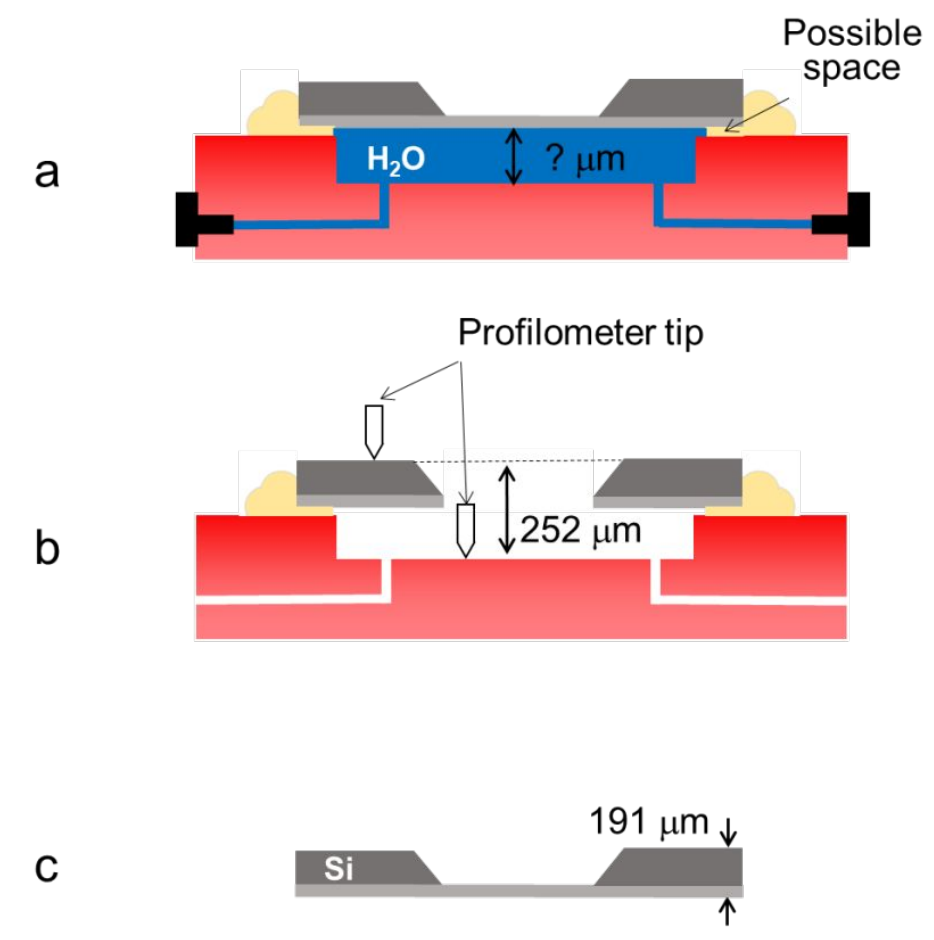

Figure S2. Determination of the accurate thickness of the water/ice layer in a liquid chamber. (a) A schematic showing the liquid chamber before SIMS testing. Some space may exist between the $\mathrm{Cu}$ block and the $\mathrm{SiN}$ membrane due to the presence of glue. (b) A schematic showing the liquid chamber after SIMS testing. The SiN membrane and the ice sample were removed by sputtering, while the rest of ice was melted and drained. A stylus profilometer was used to measure the distance from the top of the Si frame to the bottom of liquid chamber (ca. $252 \mu \mathrm{m}$ for a typical chamber). (c) Determination of the thickness of the $\mathrm{Si} / \mathrm{SiN}$ layer. The thickness of the Si frame plus SiN membrane was measured using a scanning electron microscope (ca. $191 \mu \mathrm{m}$ for a typical chamber). Therefore, the thickness of ice alone is about 252-191 $=61 \mu \mathrm{m}$ (with an assumption that under flash freezing, the volume change from water to ice in the rigid chamber can be omitted). It should be noted that some volume expansion might occur during the water-to-ice transition. Therefore, in this work, we used numbers of water molecules per Ar cluster ion as the unit to express sputter rate. Therefore, the volume expansion should not influence the value of sputter rate. 


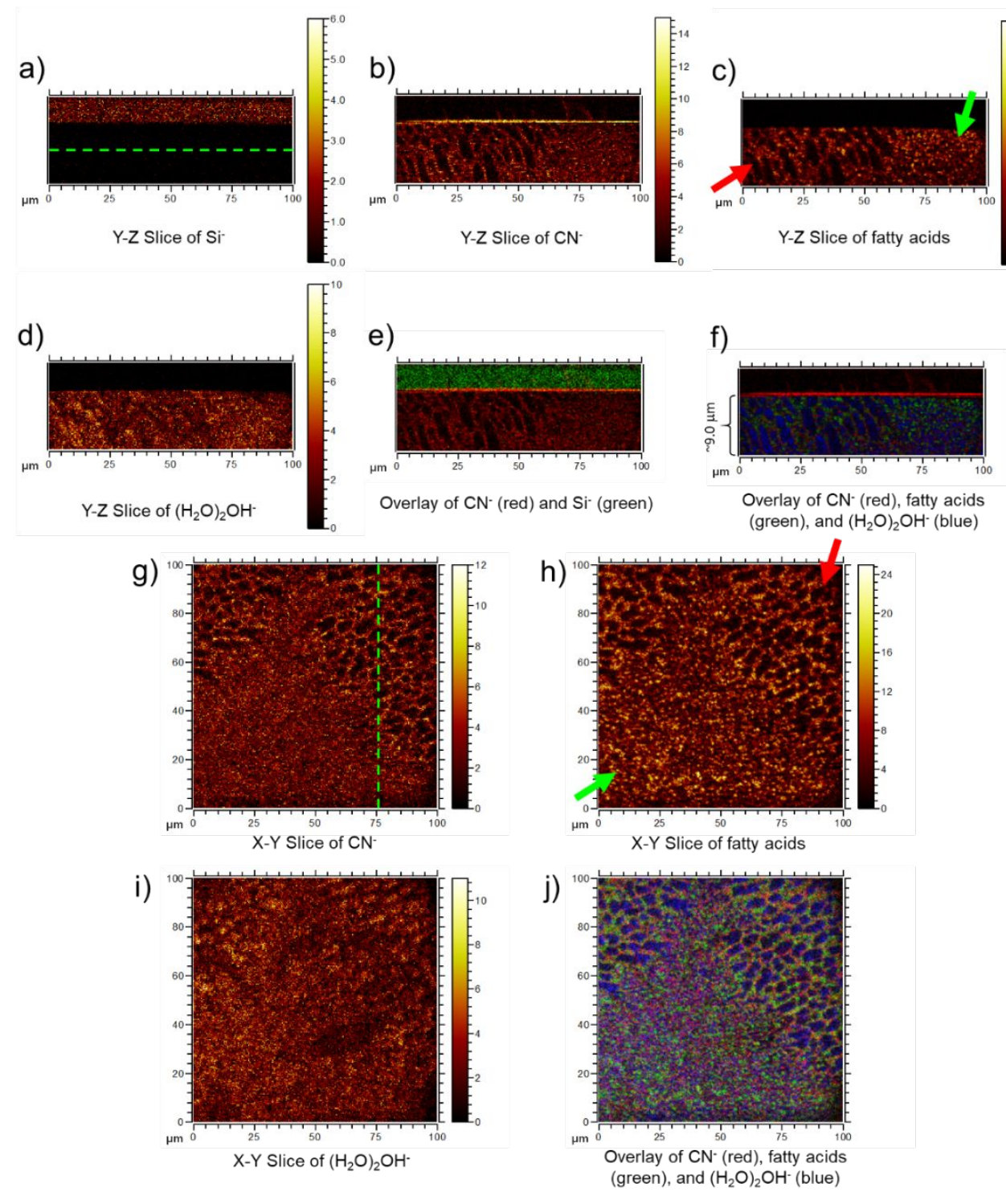

Figure S3. Negative ion images showing pseudo-3D reconstruction results of selected species in a frozen milk sample. (a) to (f) are Y-Z slices to show distributions of selected ion species along $\mathrm{Z}$-direction. The corresponding location of the $\mathrm{Y}-\mathrm{Z}$ cross-section in a X$\mathrm{Y}$ slice is denoted by a green dashed line in (g). (g) to (j) are X-Y slices, with a size of 100 $\times 100 \mu \mathrm{m}^{2}$, to show $\mathrm{X}-\mathrm{Y}$ distribution of selected species at an arbitrarily chosen depth. The corresponding location of the $\mathrm{X}-\mathrm{Y}$ cross-section in a $\mathrm{Y}-\mathrm{Z}$ slice is denoted by a green dashed line in (a). Si- (a) was shown to represent $\mathrm{SiN}$ membrane. $\mathrm{CN}^{-}(\mathrm{b}$ and $\mathrm{g}$ ) was shown to represent proteins in milk. Fatty acids species (c and h) included $\mathrm{CH}_{3}\left(\mathrm{CH}_{2}\right)_{8} \mathrm{CO}_{2}{ }^{-}$, $\mathrm{CH}_{3}\left(\mathrm{CH}_{2}\right)_{10} \mathrm{CO}_{2}^{-}, \mathrm{CH}_{3}\left(\mathrm{CH}_{2}\right)_{12} \mathrm{CO}_{2}^{-}, \mathrm{CH}_{3}\left(\mathrm{CH}_{2}\right)_{14} \mathrm{CO}_{2}^{-}$, and $\mathrm{CH}_{3}\left(\mathrm{CH}_{2}\right)_{16} \mathrm{CO}_{2}^{-}$. A water cluster signal ( $\mathrm{d}$ and i), $\left(\mathrm{H}_{2} \mathrm{O}\right)_{2} \mathrm{OH}^{-}$, is used to represent ice in the frozen milk. The thickness of milk sample measured in (a) to (f) was $\sim 9.0 \mu \mathrm{m}$ (as shown in (f)), which was estimated based on the sputter rate of ice. Red arrows in (c) and (h) denote the region with well- 
defined network structures, and green arrows denote the region with homogeneous distribution of milk contents. 